\title{
Dynamic response of full-length bonded anchor cable in rock slope
}

\author{
Nie Yong ${ }^{1}$, Zhao Yufei ${ }^{1, *}$, Du Guangjin ${ }^{2}$ \\ ${ }^{1}$ State Key Laboratory of Simulation and Regulation of Water Cycle in River Basin, China Institute of Water Resources and Hydropower \\ Research (IWHR), Beijing 100048, China \\ ${ }^{2}$ Lawa Branch of Huadian Jinsha River Upstream Hydropower Development CO.,LTD, Sichuan 610066, China
}

\begin{abstract}
The research on the anchoring mechanism of slope anchor cable under earthquake action is not in-depth. Therefore, it has become an urgent problem in engineering practice to study the seismic characteristics of slope anchoring system and then realize the optimal design of slope anchoring. In this paper, the dynamic response of a rock slope strengthened with a full-length bonded anchor cable is studied by centrifuge model test. It is found that the internal structure of the rock slope has an important effect on the length of the bonded anchor cable. The structural surface will lead to an increase of the effective anchorage length, and the stress concentration of anchor cable will occur at the structural surface. This provides some references for the optimization of traditional anchorage theory.
\end{abstract}

\section{Introduction}

In the process of water conservancy engineering construction, numerous high and steep rock slopes are widely distributed. China is a typical country with frequent earthquakes[1,2]. To prevent or reduce the problem of slope instability caused by an earthquake, seismic reinforcement must be carried out on the slope within the earthquake zone to enhance the stability of the slope.

Engineering practice shows that the slope strengthened with an anchor rod (cable) has a good seismic performance. Many scholars have found in the investigation of seismic damage of slope in earthquake that the anchor bolt (cable) supporting slope has the optimal seismic performance, and the anchored slope generally only has a local failure, which has a good seismic effect.[3]

For water conservancy and other infrastructure projects, anchor bolt (cable) reinforcement is one of the most commonly used slope reinforcement techniques, including passive mortar bolt (cable) and prestressed anchor bolt (cable).In water conservancy engineering, the combination of prestressed anchor (cable) and the full-length bonded anchor is the most common method for the protection of high and steep slopes with a height of more than $30 \mathrm{~m}$. However, due to the differences in structure, there are essential differences between the prestressed anchor and the non-prestressed full-length bonded anchor (cable) [4,5].

At present, the research on anchorage mechanism and seismic mechanism of slope under earthquake action started late. The existing research can not explain the anchoring mechanism of bolt (cable) supporting slope rationally, and the supporting theory lags behind the engineering practice for a long time[6]. Therefore, it is particularly important to study the anchoring mechanism and seismic performance of slope anchoring system under dynamic load, which will promote the understanding of the action mechanism of anchor rod (cable) under seismic action and provide a scientific theoretical basis for slope anchoring engineering.

To study the dynamic response of the bonded bolt (cable), this paper adopts the centrifuge model test and uses the full-length bonded cable to reinforce the bedding rock slope model. Under the condition of applying a dynamic load, the corresponding anchoring mechanism and dynamic response are studied by monitoring the change of slope displacement and anchor cable axial force.

\section{Test equipment and test case}

\subsection{Test equipment}

The test was carried out on the centrifuge (LXJ-4) and the centrifuge vibrator (R500B) of the China Institute of Water Resources and Hydropower Research, as shown in Figure 1. Main parameters of test equipment are shown in Table 1.

The model box adopts an aluminum alloy model box with an internal size of $750 \mathrm{~mm} \times 350 \mathrm{~mm} \times 520 \mathrm{~mm}$, as shown in the figure. After the completion of the anchoring slope model, it is put into the model box, and then the $50 \mathrm{~mm}$ high gap between the bottom of the model and the bottom layer of the model box is filled

\footnotetext{
* Corresponding author: zhaoyf@iwhr.com
} 
with cement mortar, so that the model and the model box are rigidly connected.
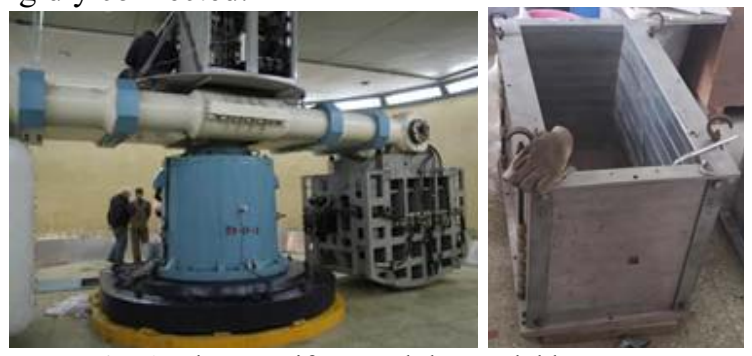

Fig. 1. The centrifuge and the model box.

Table 1. Main parameters of the experimental equipment.

\begin{tabular}{|c|c|}
\hline $\begin{array}{c}\text { LXJ-4-450g-t geotechnical } \\
\text { centrifuge }\end{array}$ & R500B shaking table \\
\hline $\begin{array}{c}\text { Maximum acceleration of } \\
\text { dynamic test centrifuge: } 60 \mathrm{~g}\end{array}$ & $\begin{array}{c}\text { Maximum vibration } \\
\text { acceleration: } 30 \mathrm{~g} \text { (horizontal), }\end{array}$ \\
\hline Rotational radius: $5.03 \mathrm{~m}$ & $\begin{array}{c}\text { Maximum vibration frequency: } \\
400 \mathrm{~Hz}\end{array}$ \\
\hline Payload: $1.5 \mathrm{t}$ & $\begin{array}{c}\text { Maximum vibration duration: } \\
3 \mathrm{~s}\end{array}$ \\
\hline $\begin{array}{c}\text { Size of the hanging basket: } \\
1.5 \mathrm{~m}^{*} 1.0 \mathrm{~m} * 1.5 \mathrm{~m}\end{array}$ & $\begin{array}{c}\text { Maximum vibration load: } \\
440 \mathrm{~kg}\end{array}$ \\
& $\begin{array}{c}\text { Vibration waveform: sine } \\
\text { wave, arbitrary seismic wave }\end{array}$ \\
\hline
\end{tabular}

\subsection{Similarity law}

Based on the technical parameters of the test platform and the main scientific problems targeted by the model test, the centrifugal acceleration of the dynamic model test was determined to be $30 \mathrm{~g}$. The similarity relationship of the model test is shown in Table 2.

Table 2. Similarity ratio of model test.

\begin{tabular}{|c|c|}
\hline Physical values & $\begin{array}{c}\text { The similarity ratio between the } \\
\text { model and the prototype }\end{array}$ \\
\hline Length $(\mathrm{L})$ & $1 / 30$ \\
\hline Area $(\mathrm{A})$ & $1 / 30^{2}$ \\
\hline Volume $(\mathrm{V})$ & $1 / 30^{3}$ \\
\hline Acceleration $(\mathrm{a})$ & $1 / 30$ \\
\hline Stress $(\sigma)$ & 1 \\
\hline Strain $(\varepsilon)$ & 1 \\
\hline Time $(\mathrm{t})$ & 30 \\
\hline
\end{tabular}

\subsection{Model design and processing}

The test model consists of two parts: slope and anchor cable. The rock slope is poured with concrete mortar. In the model, there are 3 rows and 2 rows of anchor cables, 6 in total. The specific parameters are shown in Figure 2. Cement mortar is made of 42.5 ordinary Portland cement, standard sand, and water. Through the comparison of various material parameters, the mixture ratio used in this test is cement: sand: water $=1: 3: 1$. A red copper sheet with a section size of $1 \mathrm{~mm} \times 10 \mathrm{~mm}$ is adopted to simulate the anchor cable. Red copper strip elastic modulus $108 \mathrm{GPa}$, Poisson's ratio 0.32 , density $8.9 \mathrm{~g} / \mathrm{cm}^{2}$.

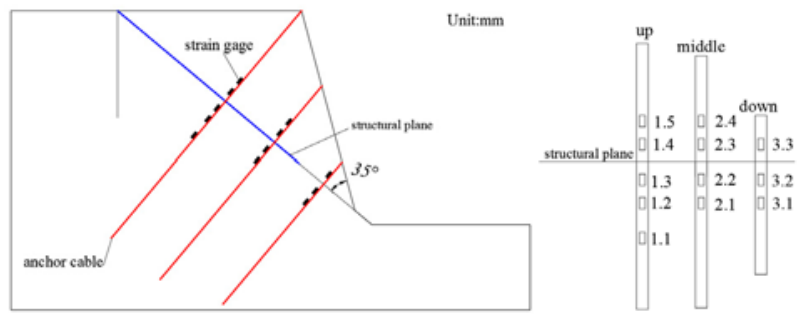

Fig. 2. Model design parameters and Strain gauge arrangement.

The strain gage is installed on the anchor cable.5, 4, and 3 strain gage are arranged on the up, middle, and down three rows of anchor cable respectively, as shown in Figure 2. The specific location is shown in Figure 2. To monitor the slope top displacement during the dynamic load process, a sensor is installed to monitor the vertical displacement.

\subsection{Test loading scheme}

To study the dynamic response law of anchor cable in rock anchored slope, the standard sine wave was used as the input of seismic wave, and the input of seismic wave was taken as the horizontal input. The dynamic load duration and period of the model test were $2.4 \mathrm{~s}$ and $0.019 \mathrm{~s}$ respectively. In this test, a total of two working conditions were designed, under the condition of different horizontal ground motion peak acceleration $(7.5 \mathrm{~g}, 15 \mathrm{~g})$ under the centrifugal acceleration of $30 \mathrm{~g}$, and the response of anchored rock slope was observed and compared.

\section{Results and Discussion}

Based on the above test scheme, the centrifugal shaking table model test of rock anchored slope with a structural surface was completed. Different types of sensors installed were used to record the time history data of the whole process of slope displacement and anchor cable axial force under dynamic load. The following is to analyze the dynamic response law of anchoring slope from the slope top displacement and the dynamic change of anchor cable axial force.

\subsection{Vertical displacement at slope top}

Figure 4 shows the time-history curve of the vertical displacement of the upper surface of the rock anchored rock slope under different horizontal seismic loads $(7.5 \mathrm{~g}$, $15 \mathrm{~g}$ ). In Figure 3, as the dynamic load is applied, the displacement of the upper slope sliding body gradually increases. At first, the rate of increase is relatively fast, then it increases slowly. When the seismic load stops being applied, the vertical displacement of the upper slide decreases gradually. Among them, it takes more than twice as long for displacement value under $15 \mathrm{~g}$ seismic load to decrease to the lowest value as it does under $7.5 \mathrm{~g}$ seismic load. Finally, after applying the dynamic load, the displacement of the slope upper part not return to its original value, and the slope has plastic deformation. 

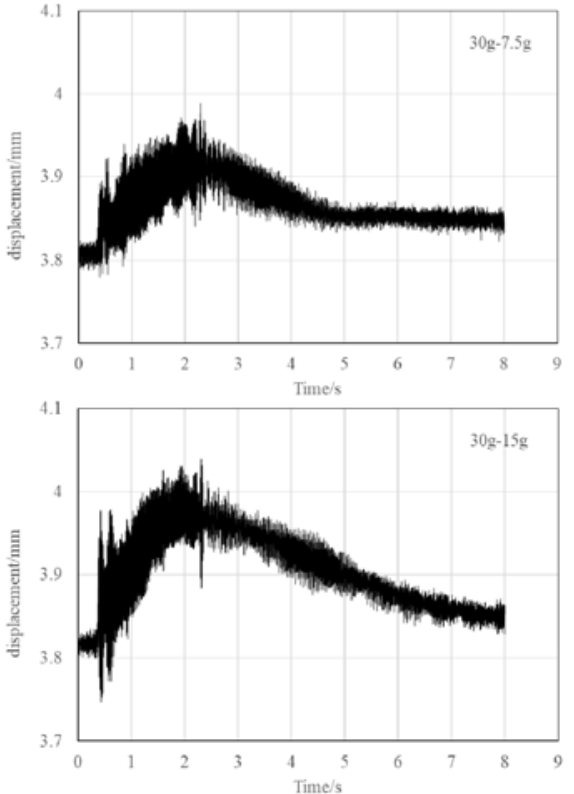

Fig. 3. Displacement of the slope (7.5g, 15g).

Figure 4 shows the displacement amplitudes of the vertical displacement time-history curves of the upper surface of the slope under two working conditions. In Figure 4, that the displacement trend of the two working conditions is the same, and the displacement of $15 \mathrm{~g}$ is generally bigger than $7.5 \mathrm{~g}$. For the displacement amplitude, when the initial amplitude is much greater than $7.5 \mathrm{~g}$ in $15 \mathrm{~g}$ earthquake, the two tend to be consistent with the application of dynamic load.

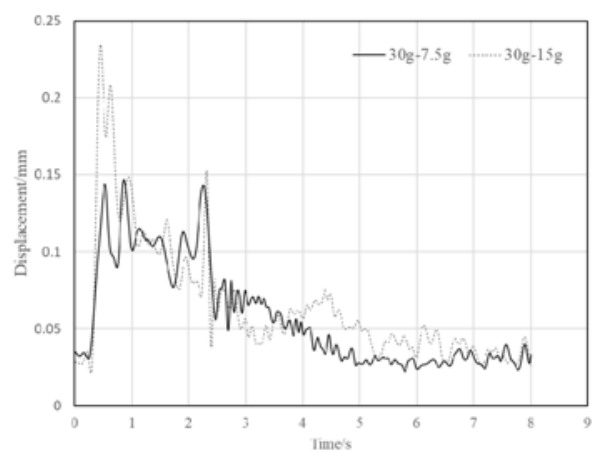

Fig. 4. Displacement amplitudes (7.5g, 15g).

\subsection{Axial force of anchor cable}

\subsubsection{Time history curve}

During the test, the strain gauge mounted on the anchor cable was used to record the axial force dynamic change of anchor cable under two conditions. Figure 5 shows the time-history curve of the anchor rope strain gauge under a working condition $(7.5 \mathrm{~g})$. It can be seen that the axial force time-history curves of anchor cable under different seismic peak acceleration conditions are generally similar. The anchor cable axial force in the uppermost layer is the largest, followed by the middle layer and the bottom layer. It is found that the anchor cable axial force with great variation in axial force is stabilized at a higher level at the initial stage of load application, and then decreases gradually. When the seismic load is over, the axial force of the anchor cable becomes 0 .

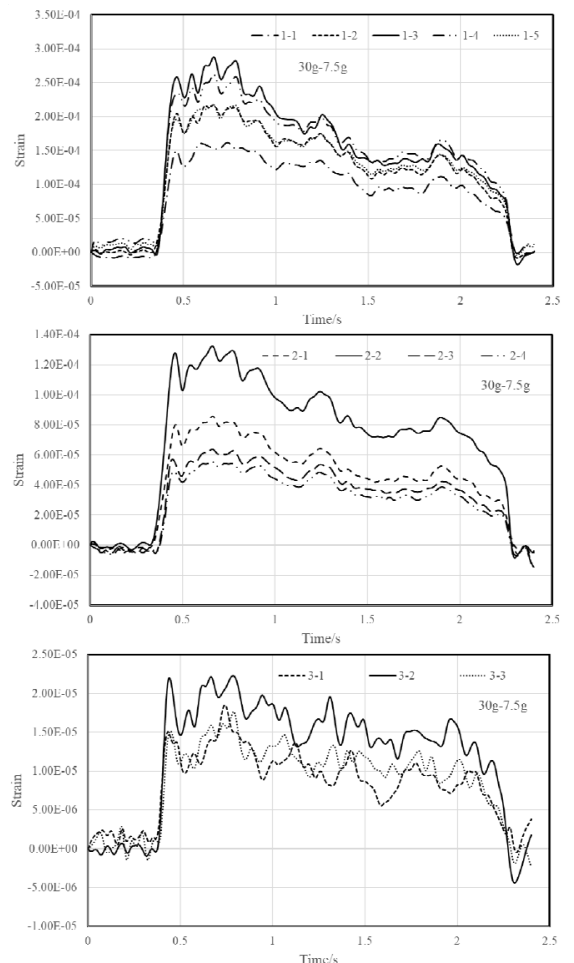

Fig. 5. The time-history curve of strain gauge data.

\subsubsection{Stress distribution on a single anchor cable}
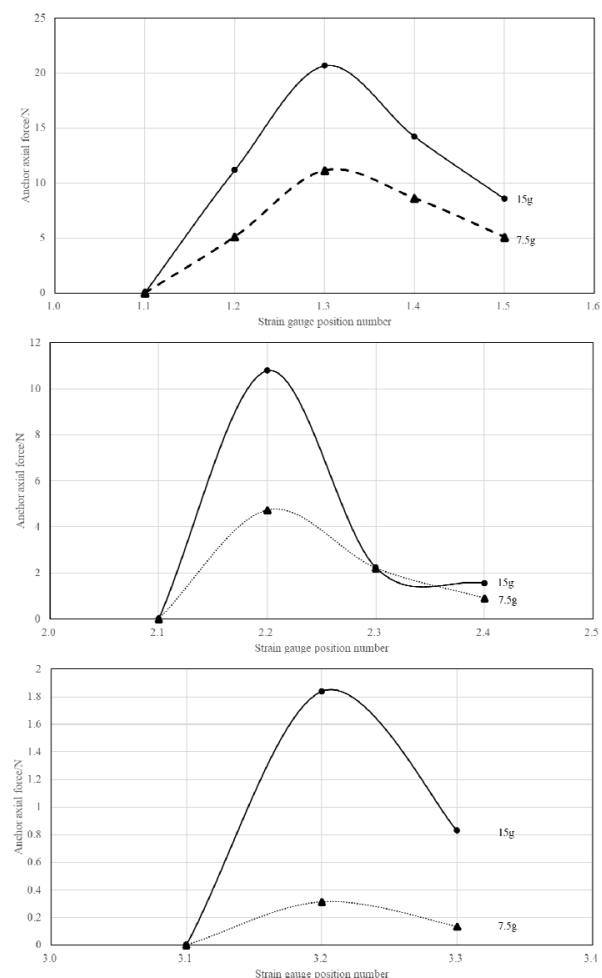

Fig. 6. Anchor cable axial forces at different positions.

Figure 6 shows the magnitude of axial forces at different positions of the different rows of anchor cable at a certain moment under dynamic load. The abscissa represents the position of strain gauge, and the ordinate 
represents the value of strain gauge at this position. It is found that the anchor cable axial force near the structural plane is larger, the farther away the structural plane is, the smaller the axial force is. And the larger the peak acceleration $(15 \mathrm{~g})$ is, the larger the influence range is, but the expansion trend is not obvious.

\subsection{Analysis of slope displacement and anchor cable axial force}

Only when the relative displacement or relative displacement trend occurs can the full-length bonded anchor cable play a supporting role. Therefore, the acting force of anchor cable under dynamic load is caused by the relative displacement or relative displacement trend of rock mass caused by the dynamic load.

For the rock anchored slope as shown in Figure 8, due to the influence of structural plane, the slope is divided into two parts: the upper and the lower rock mass. The lower rock mass is directly connected to the earth, so it can be considered that no damage will occur. The upper is connected to the lower by anchor cable and structural plane constraints.

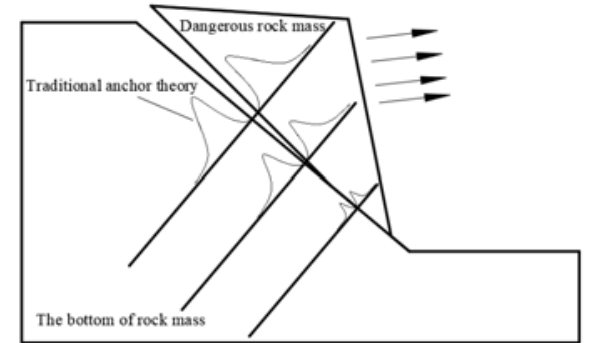

Fig. 7. Slope deformation and anchorage system action.

Through the above analysis, the most unfavorable moment of the anchoring slope under the action of dynamic load should be the moment when the relative displacement between the rock mass and the bedrock reaches its maximum value, at which time the anchor cable axial force reaches maximum. If the force of seismic on the rock mass is greater than the pulling force that the bolt can bear, the anchor cable will fail. The deformation diagram of the model under dynamic load is shown in Figure 7, which also directly explains why the upper row anchor cable axial force is the largest.

The anchor cable axial force on both sides of the structural plane can be analyzed by using the traditional anchor cable model $[7,8]$. For the traditional drawing model, the stress curve of anchor cable is shown in Figure 7 . The change curve of anchor cable axial force on both sides of the structural plane of the model generally conforms to the drawing model, as shown in Figure. 7.

\section{Conclusions}

In this paper, through the centrifuge model test, the anchoring mechanism of a full-length bonded anchor cable of rock anchored slope under dynamic load is studied, and the following conclusions are preliminarily obtained:
1.In the process of seismic action, the rock mass on the outside of the structural plane tends to move outwards relative to the rock mass on the inside of the structural plane, and the transfer process of seismic force is that the outside rock mass transfers the force to the inside rock mass through bolts and structural plane.

2. When anchoring slope with a structural surface, the effective anchoring length of full-length bonding cable is related to the structural surface, which will increase the anchoring length.

3.For traditional anchoring system design theory, the action of anchor cable axial force is simply considered. Meanwhile, the anchor length of the anchor cable is constant. In this paper, we have a new understanding of the anchoring theory of the full-length bonded cable.

\section{Acknowledgments}

This research was supported by the National Key R\&D Program of China, grant number 2018YFC0407003; National Natural Science Foundation of China, grant number 51778379.

\section{References}

1. Deng, Q.D, et al. "Active tectonics and earthquake activities in China." Earth Science Frontiers 10. SUPP (2003): 66-73.

2. $\mathrm{Xu}, \mathrm{X} . \mathrm{W}$, et al. "Coseismic reverse-and oblique-slip surface faulting generated by the $2008 \mathrm{Mw} 7.9$ Wenchuan earthquake, China." Geology 37.6 (2009): 515-518.

3. Zheng, Y.R, et al. "Engineering treatment of slope \& landslide." Beijing: China (2007).

4. Chen, A.M., Jun, S., Xin, G. "Model experiment study on the different reinforcement effects of unbonded anchor cable and full-length bonded anchor cable in rock engineering." Chinese Journal of Rock Mechanics and Engineering 24.15 (2005): 2.

5. Cheng, L.K., Zhang, P.W., Wang, F. "Several mechanical concepts for anchored structures in rock and soil." Chinese Journal of Rock Mechanics and Engineering 34.4 (2015): 668-682.

6. Zhang L.W., Li S.C. "Current State and Development of Anchorage Support for Rocks and Soils." Chinese Journal of Rock Mechanics and Engineering (2003): S1.

7. Zhang, X., Chen, SH. "Analytical solution for load transfer along anchored section of prestressed anchor cable." Rock and Soil Mechanics 36.6 (2015): 1667-1675.

8. You, CA. "Mechanical analysis on wholly grouted anchor." Chinese Journal of Rock Mechanics and Engineering 19.3 (2000): 339-341. 